\title{
OSCILLATION OF NONLINEAR DELAY DIFFERENCE EQUATIONS
}

\section{JIANCHU JIANG}

(Received 16 March 2000)

ABSTRACT. We obtain some oscillation criteria for solutions of the nonlinear delay difference equation of the form $x_{n+1}-x_{n}+p_{n} \prod_{j=1}^{m} x_{n-k_{j}}^{\alpha_{j}}=0$.

2000 Mathematics Subject Classification. 39A10.

1. Introduction. Consider the nonlinear delay difference equation

$$
x_{n+1}-x_{n}+p_{n} \prod_{j=1}^{m} x_{n-k_{j}}^{\alpha_{j}}=0, \quad n=0,1,2, \ldots
$$

where $p_{n} \geq 0, n=0,1,2, \ldots, 0 \leq k_{1} \leq k_{2} \leq \cdots \leq k_{m}$ are integers, $\alpha_{j}>0$ are rational numbers with denominator of positive odd integers for each $j=1,2, \ldots, m$ and $\sum_{j=1}^{m} \alpha_{j}=1$.

Equation (1.1) is a discrete analogue of the following first-order nonlinear delay differential equation

$$
x^{\prime}(t)+p(t) \prod_{j=1}^{m}\left[x\left(t-\tau_{j}\right)\right]^{\alpha_{j}}=0,
$$

where $p(t) \in C\left(\left[t_{0}, \infty\right),[0, \infty)\right), 0 \leq \tau_{1} \leq \tau_{2} \leq \cdots \leq \tau_{m}$, and $\alpha_{j}$ are the same as in (1.1). For (1.2), the oscillation of its solutions has been extensively studied in the literature, see, for example [2, 4, 11, 12].

When $k_{1}=k_{2}=\cdots=k_{m}=k$, (1.1) reduces to the linear delay difference equation

$$
x_{n+1}-x_{n}+p_{n} x_{n-k}=0, \quad n=0,1,2, \ldots
$$

Recently, there has been a lot of activity concerning the oscillatory behavior of (1.3). See, for example, [1, 3, 5, 6, 7, 8, 9, 10]. In particular, [7] proved that every solution of (1.3) is oscillatory provided

$$
\sum_{n=0}^{\infty}\left[\sum_{i=n}^{n+k} p_{i} \ln \left(\sum_{i=n}^{n+k} p_{i}+1-\operatorname{sign} \sum_{i=n}^{n+k} p_{i}\right)-\sum_{i=n+1}^{n+k} p_{i} \ln \left(\sum_{i=n+1}^{n+k} p_{i}+1-\operatorname{sign} \sum_{i=n+1}^{n+k} p_{i}\right)\right]=\infty
$$

Condition (1.4) improves many previous well-known results. Furthermore, (1.4) fits the case when $\sum_{i=n-k}^{n-1} p_{i}-(k /(k+1))^{k+1}$ oscillate or $\sum_{i=n-k}^{n-1} p_{i} \leq(k /(k+1))^{k+1}$. 
Our main aim in this note is to generalize condition (1.4) to (1.1).

\section{Main results}

THEOREM 2.1. Assume that

$$
\begin{aligned}
\sum_{n=0}^{\infty}\{ & \sum_{j=1}^{m} \alpha_{j} \sum_{i=n}^{n+k_{j}} p_{i} \ln \left[\sum_{j=1}^{m} \alpha_{j} \sum_{i=n}^{n+k_{j}} p_{i}+1-\operatorname{sign}\left(\sum_{j=1}^{m} \alpha_{j} \sum_{i=n}^{n+k_{j}} p_{i}\right)\right] \\
& \left.-\sum_{j=1}^{m} \alpha_{j} \sum_{i=n+1}^{n+k_{j}} p_{i} \ln \left[\sum_{j=1}^{m} \alpha_{j} \sum_{i=n+1}^{n+k_{j}} p_{i}+1-\operatorname{sign}\left(\sum_{j=1}^{m} \alpha_{j} \sum_{i=n+1}^{n+k_{j}} p_{i}\right)\right]\right\}=\infty .
\end{aligned}
$$

Then every solution of (1.1) oscillates.

Proof. Assume, by way of contradiction, that (1.1) has an eventually positive solutions $\left\{x_{n}\right\}$. Then there exists an integer $n_{1}>0$ such that $x_{n-k_{m}}>0, x_{n+1} \leq x_{n}$, $n \geq n_{1}$. We define the functions $p(t)$ and $x(t)$ as follows:

$$
p(t)=p_{n}, \quad x(t)=x_{n}+(t-n)\left(x_{n+1}-x_{n}\right), \quad n \leq t<n+1, n=0,1,2, \ldots
$$

Let $x^{\prime}(t)$ denote derivation on the right. Then

$$
\begin{gathered}
x(t)>0, \quad x^{\prime}(t) \leq 0, \quad t \geq n_{1}, \\
x^{\prime}(t)=x_{n+1}-x_{n} \text { for } n \leq t<n+1, n=0,1,2, \ldots .
\end{gathered}
$$

Hence, (1.1) can be rewritten as

$$
x^{\prime}(t)+p(t) \prod_{j=1}^{m}\left(x\left[t-k_{j}\right]\right)^{\alpha_{j}}=0, \quad t \geq 0,
$$

where here and in the sequel, $[\cdot]$ denotes the greatest integer function.

Set $\lambda(t)=-x^{\prime}(t) / x(t)$ for $t \geq n_{1}$. Then $\lambda(t) \geq 0$ for $t \geq n_{1}$, and from (2.4) we have

$$
\lambda(t)=p(t) \exp \left(\sum_{j=1}^{m} \alpha_{j} \int_{\left[t-k_{j}\right]}^{t} \lambda(s) d s\right), \quad t \geq n_{1}+k_{m},
$$

or

$$
\begin{aligned}
\lambda(t) & \sum_{j=1}^{m} \alpha_{j} \int_{t}^{\left[t+k_{j}+1\right]} p(s) d s \\
& \geq p(t)\left(\sum_{j=1}^{m} \alpha_{j} \int_{t}^{\left[t+k_{j}+1\right]} \lambda(s) d s\right) \exp \left(\sum_{j=1}^{m} \alpha_{j} \int_{\left[t-k_{j}\right]}^{t} \lambda(s) d s\right), \quad t \geq n_{1}+k_{m} .
\end{aligned}
$$

One can easily show that

$$
\phi(r) e^{x} \geq \phi(r) x+\phi(r) \ln (e r+1-\operatorname{sign} r), \quad r \geq 0, x \geq R,
$$

where $\phi(0)=0$ and $\varphi(r) \geq 0$ for $r>0$. By the definition of $p(t)$, we see that $p(t)$ is 
nonnegative and right-continuous. Therefore, it follows from

$$
\sum_{j=1}^{m} \alpha_{j} \int_{t}^{\left[t+k_{j}+1\right]} p(s) d s=0
$$

that $p(t)=0$. Applying inequalities (2.7) to the right side of (2.6), we obtain

$$
\lambda(t) \sum_{j=1}^{m} \alpha_{j} \int_{t}^{\left[t+k_{j}+1\right]} p(s) d s \geq p(t) \sum_{j=1}^{m} \alpha_{j} \int_{\left[t-k_{j}\right]}^{t} \lambda(s) d s+p(t) \ln A(t), \quad n \geq n_{1}+k_{m},
$$

where

$$
A(t)=e \sum_{j=1}^{m} \alpha_{j} \int_{t}^{\left[t+k_{j}+1\right]} p(s) d s+1-\operatorname{sign}\left(\sum_{j=1}^{m} \alpha_{j} \int_{t}^{\left[t+k_{j}+1\right]} p(s) d s\right) .
$$

Set $n_{2}=n_{1}+k_{m}$. Integrating both sides of (2.9) from $n_{2}$ to $N>n_{2}+2 k_{m}$, we have

$$
\begin{aligned}
& \sum_{j=1}^{m} \alpha_{j} \int_{n_{2}}^{N} \lambda(t) \int_{t}^{\left[t+k_{j}+1\right]} p(s) d s d t \\
& \quad \geq \sum_{j=1}^{m} \alpha_{j} \int_{n_{2}}^{N} p(t) \int_{\left[t-k_{j}\right]}^{t} \lambda(s) d s d t+\int_{n_{2}}^{N} p(t) \ln A(t) d t .
\end{aligned}
$$

Interchanging the order of integration, we get

$$
\begin{aligned}
\int_{n_{2}}^{N} p(t) \int_{\left[t-k_{j}\right]}^{t} \lambda(s) d s d t & \geq \int_{n_{2}}^{N-k_{j}} \lambda(s) \int_{s}^{\left[s+k_{j}+1\right]} p(t) d t d s \\
& =\int_{n_{2}}^{N-k_{j}} \lambda(t) \int_{t}^{\left[t+k_{j}+1\right]} p(s) d s d t
\end{aligned}
$$

Substituting this into (2.11), we have

$$
\sum_{j=1}^{m} \alpha_{j} \int_{N-k_{j}}^{N} \lambda(t) \int_{t}^{\left[t+k_{j}+1\right]} p(s) d s d t \geq \int_{n_{2}}^{N} p(t) \ln A(t) d t
$$

From (1.1) we have

$$
x_{n+1}-x_{n}+p_{n} x_{n}^{1-\alpha_{m}} x_{n-k_{m}}^{\alpha_{m}} \leq 0, \quad n \geq n_{1} .
$$

Set $y_{n}=x_{n}^{\alpha_{m}}$ for $n \geq n_{1}$. Then

$$
y_{n+1}-y_{n}+\alpha_{m} p_{n} y_{n-k_{m}} \leq 0, \quad n \geq n_{1}+k_{m} .
$$

It follows that $\alpha_{m} \sum_{i=n-k_{m}}^{n} p_{i} \leq 1$, and so

$$
\alpha_{m} \int_{t}^{\left[t+k_{m}+1\right]} p(s) d s \leq \alpha_{m} \sum_{i=[t]}^{[t]+k_{m}} p_{i} \leq 1, \quad t \geq n_{2} .
$$


Recall that $k_{1} \leq k_{2} \leq \cdots \leq k_{m}$, therefore

$$
\alpha_{j} \int_{t}^{\left[t+k_{j}+1\right]} p(s) d s \leq \frac{\alpha_{j}}{\alpha_{m}}, \quad t \geq n_{2}, j=1,2, \ldots, m .
$$

Substituting this into (2.13), we obtain

$$
\sum_{j=1}^{m} \frac{\alpha_{j}}{\alpha_{m}} \int_{N-k_{j}}^{N} \lambda(t) d t \geq \int_{n_{2}}^{N} p(t) \ln A(t) d t
$$

or

$$
\ln \left(\prod_{j=1}^{m}\left(\frac{x\left(N-k_{j}\right)}{x(N)}\right)^{\alpha_{j}}\right) \geq \alpha_{m} \int_{n_{2}}^{N} p(t) \ln A(t) d t
$$

It follows that

$$
\lim _{N \rightarrow \infty} \prod_{i=1}^{m}\left(\frac{x\left(N-k_{j}\right)}{x(N)}\right)^{\alpha_{j}} \geq \exp \left(\alpha_{m} \int_{n_{2}}^{\infty} p(t) \ln A(t) d t\right) .
$$

Let $E=\left\{n \geq n_{2} \mid p_{n}>0\right\}$. Then

$$
\begin{aligned}
\int_{n_{2}}^{\infty} p(t) \ln A(t) d t & \\
= & \sum_{n=n_{2}}^{\infty} \int_{n}^{n+1} p(t) \ln \left(e \sum_{j=1}^{m} \alpha_{j} \int_{t}^{\left[t+k_{j}+1\right]} p(s) d s+1-\operatorname{sign}\left(\sum_{j=1}^{m} \alpha_{j} \int_{t}^{\left[t+k_{j}+1\right]} p(s) d s\right)\right) d t \\
= & \sum_{n=n_{2}}^{\infty} p_{n} \int_{n}^{n+1} \ln \left(e \sum_{j=1}^{m} \alpha_{j}\left(\int_{n}^{n+k_{j}+1} p(s) d s-\int_{n}^{t} p(s) d s\right)+1\right. \\
& \left.\quad-\operatorname{sign}\left(\sum_{j=1}^{m} \alpha_{j}\left(\int_{n}^{n+k_{j}+1} p(s) d s-\int_{n}^{t} p(s) d s\right)\right)\right) d t \\
= & \sum_{n=n_{2}}^{\infty} p_{n} \int_{n}^{n+1} \ln \left(e \sum_{j=1}^{m} \alpha_{j}\left(\sum_{i=n}^{n+k_{j}} p_{i}-p_{n}(t-n)\right)+1\right. \\
& \quad-\operatorname{sign}\left(\sum_{j=1}^{m} \alpha_{j}\left(\sum_{i=n}^{n+k_{j}} p_{i}-p_{n}(t-n)\right)\right) d t \\
= & \sum_{E} p_{n} \int_{n}^{n+1} \ln \left(e \sum_{j=1}^{m} \alpha_{j}\left(\sum_{i=n}^{n+k_{j}} p_{i}-p_{n}(t-n)\right)\right) d t
\end{aligned}
$$




$$
\begin{aligned}
&=\sum_{E}(\left(\sum_{j=1}^{m} \alpha_{j} \sum_{i=n}^{n+k_{j}} p_{i} \ln \left(\sum_{j=1}^{m} \alpha_{j} \sum_{i=n}^{n+k_{j}} p_{i}\right)\right) \\
&\left.-\sum_{j=1}^{m} \alpha_{j} \sum_{i=n+1}^{n+k_{j}} p_{i} \ln \left(\sum_{j=1}^{m} \alpha_{j} \sum_{i=n+1}^{n+k_{j}} p_{i}+1-\operatorname{sign}\left(\sum_{j=1}^{m} \alpha_{j} \sum_{i=n+1}^{n+k_{j}} p_{i}\right)\right)\right) \\
&=\sum_{n=n_{2}}\left(\sum_{j=1}^{m} \alpha_{j} \sum_{i=n}^{n+k_{j}} p_{i} \ln \left(\sum_{j=1}^{m} \alpha_{j} \sum_{i=n}^{n+k_{j}} p_{i}+1-\operatorname{sign}\left(\sum_{j=1}^{m} \alpha_{j} \sum_{i=n}^{n+k_{j}} p_{i}\right)\right)\right. \\
& \quad-\sum_{j=1}^{m} \alpha_{j} \sum_{i=n+1}^{n+k_{j}} p_{i} \ln \left(\sum_{j=1}^{m} \alpha_{j} \sum_{i=n+1}^{n+k_{j}} p_{i}+1-\operatorname{sign}\left(\sum_{j=1}^{m} \alpha_{j} \sum_{i=n+1}^{n+k_{j}} p_{i}\right)\right) .
\end{aligned}
$$

From (2.1) and (2.20), we have

$$
\lim _{N \rightarrow \infty} \prod_{j=1}^{m}\left(\frac{x\left(N-k_{j}\right)}{x(N)}\right)^{\alpha_{j}}=\infty
$$

On the other hand, it follows from (2.1) that

$$
\limsup _{n \rightarrow \infty} p_{n}>0
$$

By (2.15), we have

$$
\limsup _{n \rightarrow \infty} p_{n} \leq \limsup _{n \rightarrow \infty} \frac{y_{n}}{\alpha_{m} y_{n-k_{m}}}=\frac{1}{\alpha_{m}} \frac{1}{\liminf _{n \rightarrow \infty}\left(y_{n-k_{m}} / y_{n}\right)},
$$

which, together with (2.23) yields

$$
\liminf _{n \rightarrow \infty} \frac{y_{n-k_{m}}}{y_{n}}<\infty
$$

that is,

$$
\liminf _{N \rightarrow \infty}\left(\frac{x\left(N-k_{m}\right)}{x(N)}\right)^{\alpha_{m}}<\infty,
$$

and so

$$
\liminf _{N \rightarrow \infty} \prod_{j=1}^{m}\left(\frac{x\left(N-k_{j}\right)}{x(N)}\right)^{\alpha_{j}} \leq \liminf _{N \rightarrow \infty} \frac{x\left(N-k_{m}\right)}{x(N)}<\infty,
$$

which contradicts (2.22) and so the proof is completed. 
From Theorem 2.1 we have immediately.

COROLLARY 2.2. Assume that there exists an integer $N \geq 0$ such that

$$
\sum_{j=1}^{m} \alpha_{j} \sum_{i=n}^{n+k_{j}} p_{i}>0 \quad \text { for } n \geq N
$$

and that

$$
\sum_{n=N}^{\infty}\left[\sum_{j=1}^{m} \alpha_{j} \sum_{j=n}^{n+k_{j}} p_{i} \ln \left(\sum_{j=1}^{m} \alpha_{j} \sum_{i=n}^{n+k_{j}} p_{i}\right)-\sum_{j=1}^{m} \alpha_{j} \sum_{i=n+1}^{n+k_{j}} p_{i} \ln \left(\sum_{j=1}^{m} \alpha_{j} \sum_{i=n+1}^{n+k_{i}} p_{i}\right)\right]=\infty .
$$

Then every solution of (1.1) oscillates.

Clearly, when $k_{1}=k_{2}=\cdots=k_{m}$, condition (2.1) reduces to (1.4).

ACKNOWLEDGEMENT. This work was supported by the Science Foundation of Hunan Educational Committee of China.

\section{REFERENCES}

[1] L. H. Erbe and B. G. Zhang, Oscillation of discrete analogues of delay equations, Differential Integral Equations 2 (1989), no. 3, 300-309. MR 90a:39001. Zbl 723.39004.

[2] R. G. Koplatadze and T. A. Chanturia, Oscillating and monotone solutions of first-order differential equations with deviating argument, Differencial'nye Uravnenija 18 (1982), no. 8, 1463-1465 (Russian). MR 83k:34069. Zbl 0496.34044.

[3] G. Ladas, C. G. Philos, and Y. G. Sficas, Sharp conditions for the oscillation of delay difference equations, J. Appl. Math. Simulation 2 (1989), no. 2, 101-111. MR 90g:39004. Zbl 685.39004.

[4] G. Ladas and I. P. Stavroulakis, Oscillations caused by several retarded and advanced arguments, J. Differential Equations 44 (1982), no. 1, 134-152. MR 83e:34104. Zbl 477.34050.

[5] I. P. Stavroulakis, Oscillations of delay difference equations, Comput. Math. Appl. 29 (1995), no. 7, 83-88. MR 96f:39007. Zbl 832.39002.

[6] X. H. Tang, Oscillations of delay difference equations with variable coefficients, J. Central South Univ. of Technology 29 (1998), 287-288 (Chinese).

[7] X. H. Tang and J. S. Yu, A further result on the oscillation of delay difference equations, Comput. Math. Appl. 38 (1999), no. 11-12, 229-237. MR 2000m:39035.

[8] - Oscillation of delay difference equation, Comput. Math. Appl. 37 (1999), no. 7, 11-20. MR 2000c:39010. Zbl 937.39012.

[9] __ Oscillations of delay difference equations, Hokkaido Math. J. 29 (2000), no. 1, 213-228. MR 2001a:39024. Zbl 958.39015.

[10] _ Oscillations of delay difference equations in a critical state, Appl. Math. Lett. 13 (2000), no. 2, 9-15. MR 2000m:39034.

[11] J. J. Wei, On oscillation criteria for solutions of linear differential equations with deviating arguments, Math. Practice Theory (1988), no. 3, 9-19 (Chinese). MR 89j:34107.

[12] J. S. Yu, First-order nonlinear differential inequalities with deviating argument, Acta Math. Sinica 33 (1990), no. 2, 152-159. MR 91d:34069. Zbl 714.34109.

JiAnChu JiAng: DePartment of MAThematics, Loudi Teachers' COllege, LOUdi, HunAN 417000, CHINA

E-mail address: 1djjc@mai 1.1d.hn.cn 


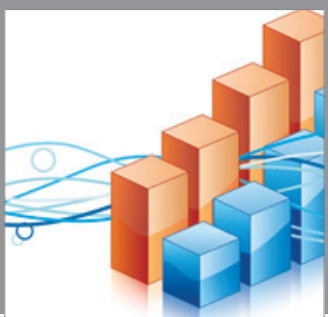

Advances in

Operations Research

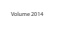

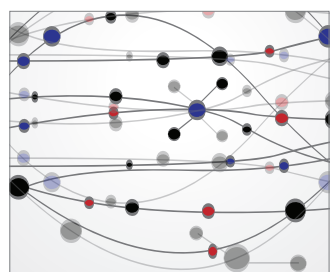

\section{The Scientific} World Journal
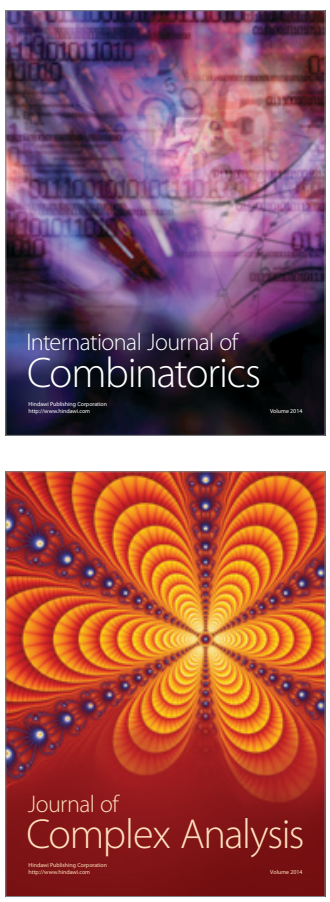

International Journal of

Mathematics and

Mathematical

Sciences
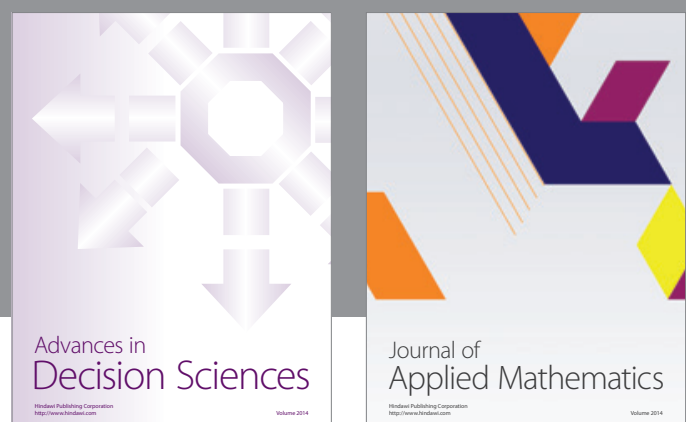

Journal of

Applied Mathematics
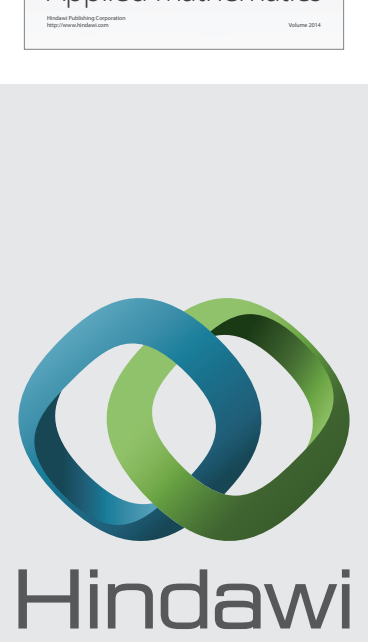

Submit your manuscripts at http://www.hindawi.com
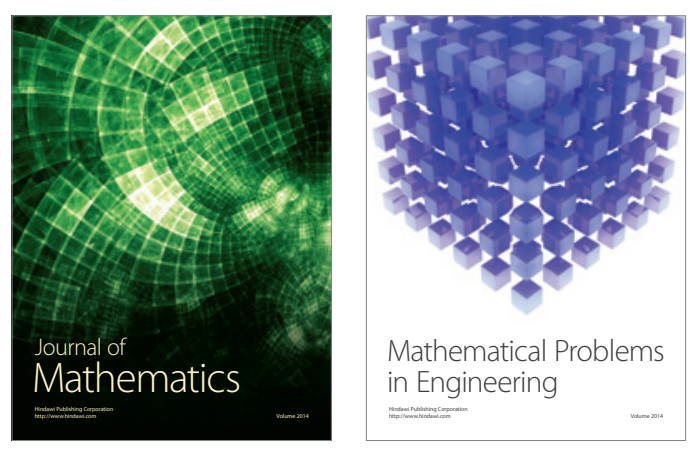

Mathematical Problems in Engineering
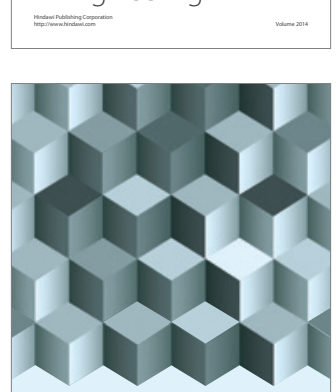

Journal of

Function Spaces
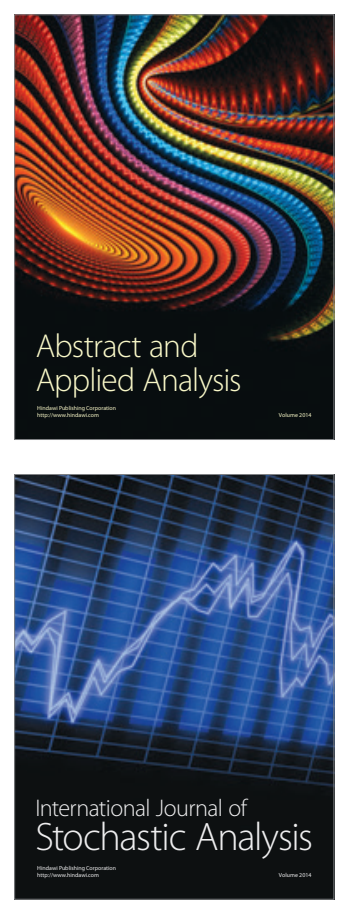

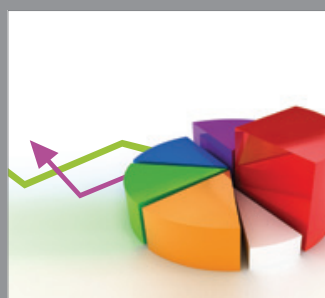

ournal of

Probability and Statistics

Promensencen
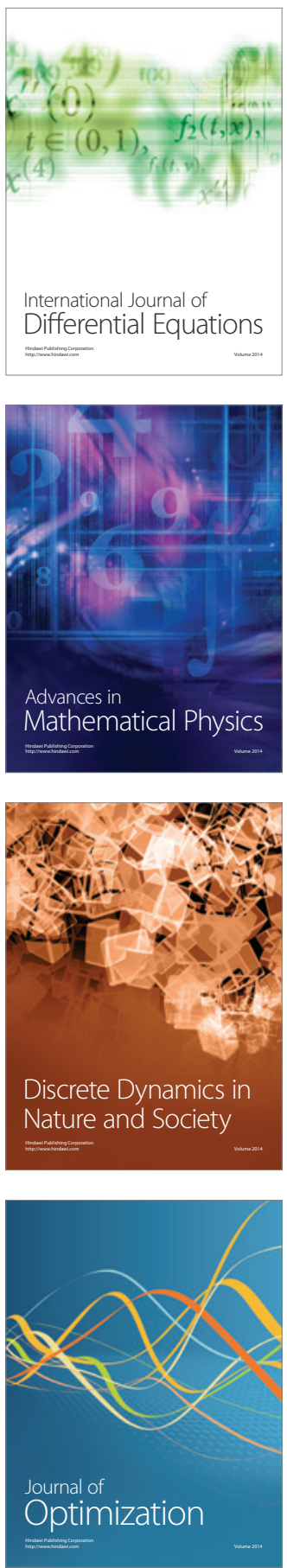